\title{
COMPARING THE SECONDARY STABILITY OF DENTAL IMPLANTS: IMMEDIATE GRADUAL LOADING VERSUS EARLY LOADING PROTOCOL IN THE POSTERIOR MAXILLA
}

\author{
Elhassan Mohammed Mossad*, Mohammed Kashaba** and Hesham Abd Elhakam***
}

\begin{abstract}
Introduction: Loading of dental implants has been performed in different protocols to achieve a high success rate and to decrease oral handicap timing. Many factors can contribute to the proper selection of loading protocol.

Objectives: This study evaluated the effect of early and immediate gradual loading protocols on the secondary stability of dental implants, which was measured by resonance frequency analysis.

Material and Methods: Ten patients each received two implants in the posterior maxillary region. At one side implants were loaded by progressive gradual loading using healing caps while the other side implants were loaded by early loading protocol. The secondary stability of dental implants was measured 6 weeks after implantation day using resonance frequency analysis.

Results: There was no statistically significant difference between (Progressive loading protocol) and (early loading protocol) groups where $(\mathrm{p}=0.863)$. The highest mean value was found in (Progressive loading protocol) $(77.86 \pm 15.74)$, while the lowest mean value was found in (early loading protocol) $(76.29 \pm 17.63)$.

Conclusion : Trans mucosal healing is possible in the posterior maxilla with a very high success rate, especially if the implant was inserted with high primary stability. However, strict oral hygiene measure is mandatory to avoid plaque accumulation which may lead to mucositis. Progressive loading with a healing cap is not of a great benefit to the patients in terms of bone training, and it might cause pain while replacing healing caps.
\end{abstract}

\section{INTRODUCTION}

The most conventional and known protocol for the successful osseointegrated dental implants includes the employment of a biocompatible material, conservative atraumatic surgical approach, and a load-free healing period minimum of three months. The goal of this approach is to achieve a stable healing environment, which results in

\footnotetext{
* Department of Oral and Maxillofacial Surgery Cairo University

** Assistant Professor, Department of Oral and Maxillofacial Surgery Cairo University

*** Professor, Department of Oral and Maxillofacial Surgery Cairo University
} 
successful osseointegration of dental implants. The most critical prerequisite for osseointegration is the load-free period, delayed loading of 6 months in maxilla and 3 months in the mandible were based by Branemark et al., and were never experimentally proved .while Henry and Rosenberg in 1994 stated that extended healing periods are not mandatory, but it is just a therapeutic reserve. This strict stressfree protocol that achieved very high success rate achieved by submerging of the dental implant under the gums, and no temporary restoration nor gingival former should be placed to prevent any loading of the fixture. Even in edentulous patients, a lag period of 2 weeks after the implant placement was mandatory before the construction of a temporary denture (Brånemark et al., 1969, 1977; Henry \& Rosenberg, n.d.; Johansson, C.; Albrektsson, n.d.)

Szmukler (2000) conducted a review of the conventional delayed loading protocol, which covered the majority of the early author's contribution to this field. Within this review, it was concluded that a delay in loading was due to non-optimized implant design or surfaces, as well as non-optimized prosthetic treatment protocols. A variety of Literature exists in the field of loading protocols, and thus cannot all be discussed here. Key experiments by Isidor have shown a soft lowdensity bone surrounding the implants with loss in its mass due to disuse atrophy. Disuse atrophy was a result of the unloaded implants, which demonstrates the necessity of alternative protocol measures to preserve the bone in implant patients. (Isidor, 1996; Moncler, Salama, Reingewirtz, \& Dubruille, 2013).

An alternative procedure that can be used to preserve bone in patients is that of a gradual loading protocol. Evidence to support this view comes from Roberts (1987), Mish (1998), and Ghoveizi (2013). Collectively these authors were able to demonstrate the effectiveness of the use of this protocol. Adapting this method led to benefits such as increased pre -implant bone density, the minimization of bone loss, and a decrease in healing time. Appleton (2005) was able to achieve gradual progressive loading using acrylic crowns with a gradual increase in its occlusal height after 6 weeks from the surgery time. The formation of more dense, and increasingly organized lamellar bone was put forward as an explanation to the reasons behind the reduction in healing time (Appleton, Nummikoski, Pigno, Cronin, \& Chung, 2005; Ghoveizi, Alikhasi, Siadat, Siadat, \& Sorouri, 2013; Misch, Hoar, Beck, Hazen, \& Misch, 1998; Roberts, 1988)

In 1980 the concept of progressive loading was launched based on empirical data supporting the idea that continuous gradual stimulation will permit bone to mature and grow denser and improve in quality (Misch 1999b). The Superior density results in superior strength, and thus the capability to tolerate higher forces and allows long term successful implant prosthetic treatment (Skalak 1983; Roberts et al. 1987; Rice et al. 1988). Roberts et al. described progressive loading protocols that control the load falling on a dental implant by adjusting the size of the occlusal table, occlusal contacts, the elimination of cantilevers, and the consistency of the diet (Misch et al., 1998; Roberts, 1988; Skalak, 1983).

According to Frost's "mechanostat theory, The improvement of bone density around implants is a result of the dynamic relationship between loading and positive bone modeling response (Frost, 1983; Wu, Guise, Frost, \& Mitchell, 1978). Also, he suggested that bone remodeling is triggered at a particular strain threshold, whereas bone hypertrophy could result when the amount of load exceeds this threshold.

Several animal studies which examined the bone reaction surrounding dental implants exposed to static load informed higher bone-to-implant contact and bone density around loaded than load free sites (Akin-Nergiz, Nergiz, Schulz, Arpak, \& Niedermeier, 1998; Gotfredsen, Berglundh, \& Lindhe, 2001; Melsen \& Lang, 2001; “Titanium 
implants and lateral forces. An experimental study with sheep).

In 1973 by Cameron et al. introduced the concept of threshold micromovement. He classified the movements into micromovements that do not affect bone ingrowth inside the titanium implant surface and macro-movements that resulted in fibrous integration. Also in a clinical study on endodontic implants carried by Maniatopoulos et al. in 1986 confirmed that there is the bone can tolerate a threshold of micro-movements without fibrous encapsulation of the fixture. (H. Cameron, Macnab, \& Pilliar, 1972; H. U. Cameron, Pilliar, \& MacNab, 1973; Maniatopoulos, Pilliar, \& Smith, 1986).

There appears to be a sense of uncertainty amongst the scientific community with regards to whether immediate gradual loading is a better choice of protocol. It is, therefore, necessary to conduct a clinical trial to find a simple treatment protocol that may shorten the healing period and reduces bone loss after implant placement. The aim of this study to provide additional evidence that gradual loading protocol may offer benefits, such as decreased healing time and the preservation of bone. Implications indicate that this may prevent thousands of unsuccessful implant treatments each year. Furthermore, It also eliminates the debate about the exact time to load the implant, thus reducing the time needed to carry out the treatment. Evidently, this will lead to advantages for both the practicing Doctor and the patient.

This study hypothesizes that immediate gradual loading using gingival formers is superior to both conventional and early loading protocols in improving bone quality and thus reducing the healing time.

\section{MATERIALS AND METHODS}

\section{Ethical approval}

Research Ethics Committee, Faculty of Oral and Dental Medicine - Cairo University
Ethical approval number: 150408

Ethical approval date: 21/4/2014

\section{Patient Enrollment}

All patients involved in this study provided their informed consent prior to treatment.

A split-mouth design study included randomly selected patients from the Outpatient Clinic of the oral and maxillofacial department of Cairo University, Egypt. Patients were included in the study according to the following criteria: 1) Patients with bilateral Partial or complete edentulous areas in maxillary premolar/molar region; 2) Free medical history; 3) Age group 25-55 years old; 4) Enough bone quantity; 5) Enough keratinized mucosa.

Exclusion criteria were:1) Irradiated bone (radiotherapy); 2) Systemic disease; 3) smoking habit; 4) Patients undergoing long-term immunosuppressive therapy; 5) Atrophic ridges. Once being registered in the study, the patients were randomly allocated to one of the two treatment groups.

\section{Presurgical preparation}

Cone beam CT scans were done by the radiologist for all of the patients to determine bone quantity. Also, additional silicon impression material and fast sitting plaster were used for construction of study cast to construct radiograph stent.

Templates were made of $1 \mathrm{~mm}$ clear shim using a clear acrylic sheet (Biostark, Great Lakes Orthodontics) over the cast model. Then trimming of the excess plastic, to allow easy placement and removal of the templates from the patient's mouth. Then, a hole drilled through the template at the central fossae of the future restoration.

\section{Surgical Protocol}

Before the surgery, the patients were given 1000 $\mathrm{mg}$ of amoxicillin trihydrate and were asked to rinse with Chlorhexidine mouth wash. Also, Patient 
lips and check were wiped by cotton roll socked in Chlorhexidine.

Anesthesia used was 2\% Xylocaine with epinephrine 1:100,000. (Infiltration on the buccal side: The needle is inserted at the height of the mucobuccal fold at a 45 degree angle. while palatal injection: The needle is inserted roughly 5 to $10 \mathrm{~mm}$ palatal to the center of the ridge).

A crestal incision was located at the center of the ridge or slightly palatal using $15 \mathrm{c}$ blades, then Fullthickness mucoperiosteal flap reflection using a sharp periosteal elevator was done. The radiograph stent was placed inside the patient mouth, and a round bur was used to mark the position of the implants on the ridge.

Implant sites were then prepared under copious irrigation according to the user manual to receive a 4.2 Bio3 progressive dental implant. Surgical drills of increasing diameters were used till the final drill of diameter of $3.7 \mathrm{~mm}$ and $12.5 \mathrm{~mm}$ in length

Initially Implant will be inserted by hand as deep as possible afterward torque wrench will be used to place the implant in the final position $1 \mathrm{~mm}$ subcrestally.

Afterwards, internal decontamination of the implants were done by Chlorhexidine gel placement into the implant cavity. Then $2 \mathrm{~mm}$ height healing cap

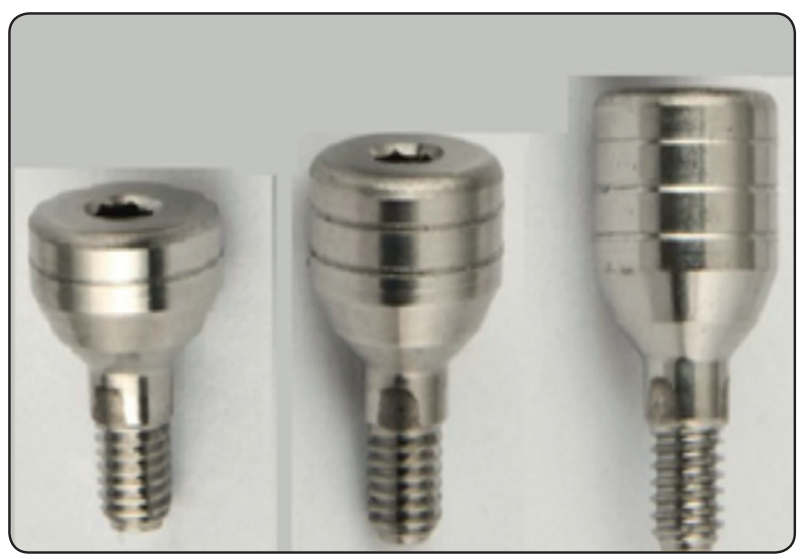

For immediate gradual loading group (at surgery time group A received $2 \mathrm{~mm}$ height gingival former which was swapped by, $3 \mathrm{~mm}$ and finally $4 \mathrm{~mm}$ ). was screwed to the implants and the mucoperiosteal flaps were sutured using 3- 0 vicryl sutures.

N.B. Both implants of the study were placed in the same surgery. Then the Patient finally rinsed with Chlorhexidine mouth wash.

\section{Post-operative care}

Antibiotic was administrated post-operatively for a week, amoxicillin trihydrate and potassium clavulanate $1 \mathrm{gm}$ every 12 hours (Augmenten $1 \mathrm{gm}$, GlaxoSmithKline, $\mathrm{UK}^{\circledR}$ ) and ibuprofen $600 \mathrm{mg}$ every 12 hours (Brufen $600 \mathrm{mg}$, Abbott, US ${ }^{\circledR}$ )

\section{Follow-up Appointments}

For the progressive loading group (group A), $2 \mathrm{~mm}$ height gingival former was screwed to the implant using Hex drive during surgery. The gingival formers were replaced every two weeks by consequent larger size gingival former (at surgery time group A received $2 \mathrm{~mm}$ height gingival former which was swapped by, $3 \mathrm{~mm}$ and finally $4 \mathrm{~mm}$ ).

Resonance frequency analysis was used to measure the secondary stability of the implants at the $6^{\text {th }}$ week after the surgery.

For the early loading group (group B). The implants remain with the $2 \mathrm{~mm}$ height gingival former from the surgery time till loading time six weeks later.

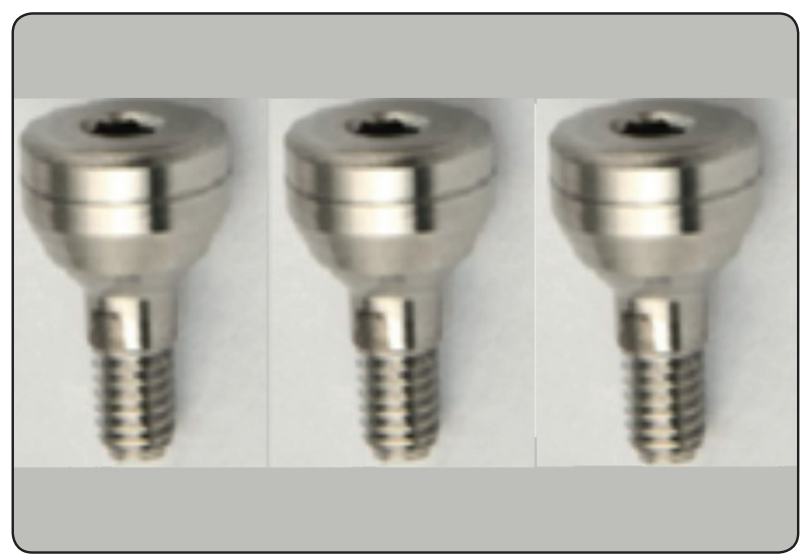

For the early loading group (group B). The implants remain with the $2 \mathrm{~mm}$ height gingival former from the surgery time till loading time six weeks later. 


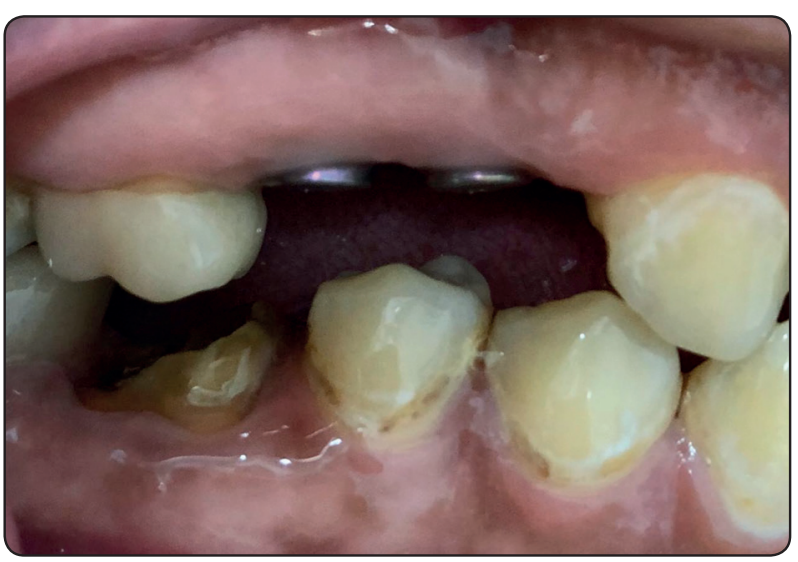

Both groups with $2 \mathrm{~mm}$ gingival formers

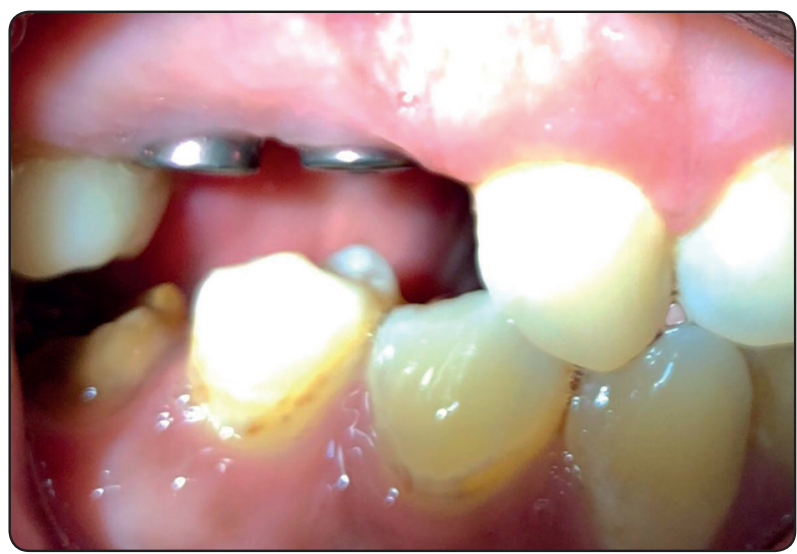

Group A received $3 \mathrm{~mm}$ gingival former while group B had the $2 \mathrm{~mm}$ gingival former

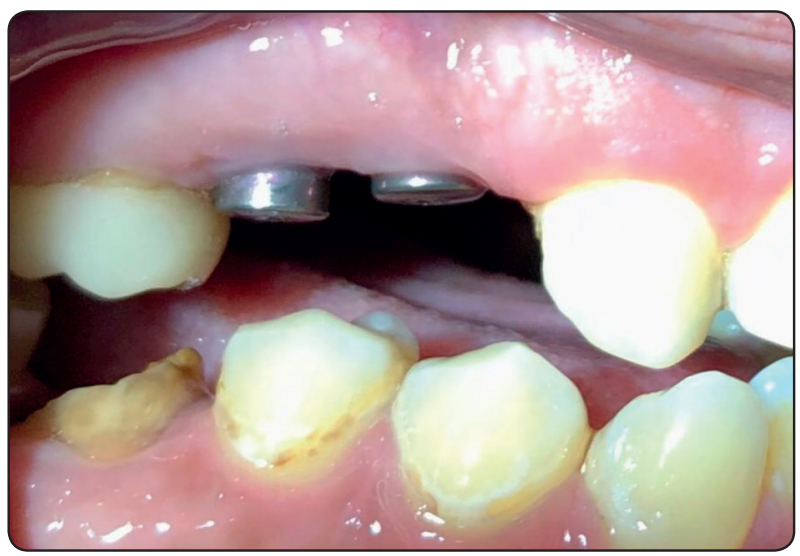

Group A recived $4 \mathrm{~mm}$ gingival former while group B is with $2 \mathrm{~mm}$ gingival former

\section{RESULTS}

Eleven patients (all were females age group from 40 to 63 years - mean $51.9 \pm 14.7)$ received 22 identical tapered implants $(4.2 \mathrm{~mm}$ in diameter and $11.5 \mathrm{~mm}$ length) in the posterior maxilla. Differences in the mean primary stability (ISQ values) between group A (mean ISQ 71.80) and group B (mean ISQ $72.60)$ were not statistically significant $(\mathrm{p}=0.826)$ as shown in table 1.

Table (1): The mean, standard deviation (SD) values of different groups.

\begin{tabular}{|c|c|c|}
\hline \multirow{2}{*}{ Variables } & \multicolumn{2}{|c|}{ Secondary stability } \\
\cline { 2 - 3 } & Mean & SD \\
\hline Group A & $71.80^{\text {a }}$ & 6.30 \\
\hline Group B & $72.60^{\text {a }}$ & 4.72 \\
\hline & \multicolumn{2}{|c|}{$\mathbf{0 . 8 2 6 n s}$} \\
\hline
\end{tabular}

Superscripts with different capital letters indicate statistically significance difference within the same row. *; significant ( $p \leq 0.05) \quad n s ;$ non-significant ( $p>0.05)$.

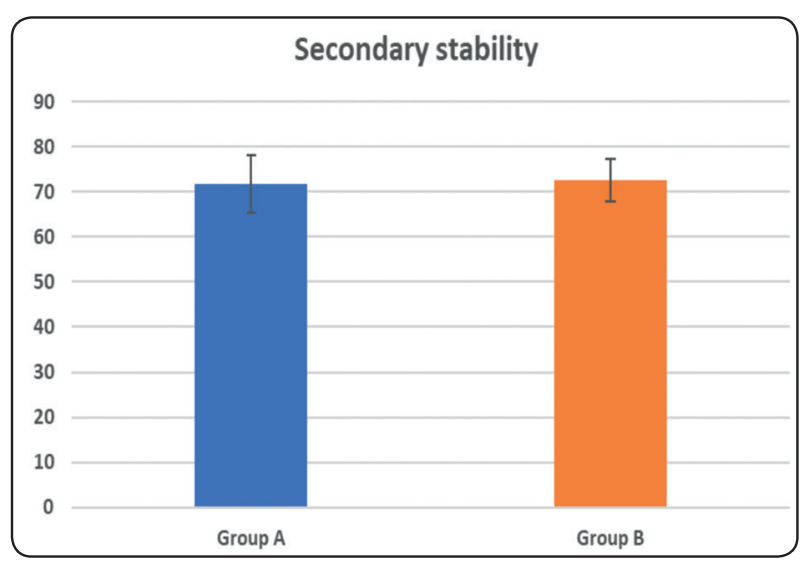

Fig. (1): Bar chart representing effect of time on secondary stability

For the progressive loading group, the highest secondary stability ISQ value was 99 and the lowest was 67 , while the early loading group's highest secondary stability ISq value was 99 and the lowest was 56 .

The mean and standard deviation values were calculated for each group in each test. Data were 
explored for normality using Kolmogorov-Smirnov and Shapiro-Wilk tests and showed parametric (normal) distribution.

Independent sample t-test was used to compare between two groups in non-related samples.

The significance level was set at $\mathrm{P} \leq 0.05$. Statistical analysis was performed with IBM® SPSS ${ }^{\circledR}$ Statistics Version 20 for Windows.

\section{Test for normality}

The results of Shapiro-Wilk test for Group A and Group B showed normal distribution pattern at 95\% confidence $(\mathrm{W}-$ Statistic $=0.928, \mathrm{P}$-value $=0.585)$ and $(\mathrm{W}-$ Statistic $=0.908, \mathrm{P}$-value $=0.457)$ respectively.

\section{Secondary stability:}

There was no statistically significant difference between (Group A) and (Group B) where ( $p=0.826)$.

The highest mean value was found in (Group B), the lowest mean value found in (Group A).

For the early loading group, mild mucositis was present during the removal of the $2 \mathrm{~mm}$ gingival former, and it was noticed more plaque accumulation in comparison to the progressively loaded group. For the progressive loading group, three patients suffered from pain every time we replaced the gingival formers, so local anesthesia was administrated to relief the pain every time we changed the healing caps. Also, one of these patients had a painful gingival swelling around one of the progressively loaded healing caps that was relieved by the administration of antibiotics and mouth wash for one week.

All gingival formers were stable without loosening except for one patient who was excluded from this study. All implants in this study were osseointegrated, and no implants were lost. Also, none of the implants was spinner during torquing of the abutments at $35 \mathrm{~N} \mathrm{~cm}$ during the final cementation of the final prosthesis. Also, neither local nor systemic adverse side effects occurred in any surgery during this study

\section{DISCUSSION}

The aim of this study was to compare the secondary stability of the implants loaded with progressive loading protocol with early loading protocol. In this study, we tried to diminish all of the errors influencing implant placement and stability readings in order to apply our attention to the impact of loading protocol on secondary stability. Identical implants in diameter $(4.2 \mathrm{~mm})$, length $(11.5 \mathrm{~mm})$, design were used, and all of the implants were inserted at the same bone level. Implant site choice (posterior maxillary area) and surgical preparation technique were standardized to reduce the errors.

Resonance frequency analysis (isq) is a noninvasive and accurate method that was chosen as a to measure implant's secondary stability. Abrahamsson declared that a resonance frequency value does not reflect histological results, but it can determine the appropriate loading time (Abrahamsson, Linder, \& Lang, 2009; Huwiler, Pjetursson, Bosshardt, Salvi, \& Lang, 2007).

In this split-mouth study design, each side received one implant and both went under transmucosal healing using gingival former, and both were implanted in the same visit. Eating will allow food to come in contact with healing caps, which transmits the load to the bone surrounding the implants in both groups. According Misch to continuous gradual stimulation will permit bone to mature and grow denser and improve in quality this might be a reason for no significant difference in secondary stability measurements and stable bone level as both implants were loaded. Also, high isq values, and all implants withstood the abutment tourqing at $35 \mathrm{Ncm}$ without spinning may reflect the improvement of bone quality.

On the contrary to this, the description of Roberts et al. for progressive loading protocols which is the control of the load falling on a dental implant by adjusting the size of the occlusal table, occlusal contacts, and the consistency of the diet. In this study occlusal table was fixed as the gingival formers are 
of a fixed diameter of $4 \mathrm{~mm}$, they were totally out of occlusion, and no dietary recommendation was given to the patients, so no significant difference in secondary stability measurements may be due to inadequate bone loading. According to Frost's "mechanostat theory, The improvement of bone density around implants is a result of the dynamic relationship between loading and positive bone modeling response (Frost 1983, 1987). Also, he suggested that bone remodeling is triggered at a particular strain threshold, whereas bone hypertrophy could result when the amount of load exceeds this threshold. Maybe in this study, there was not enough load that could stimulate positive bone modeling. Also, all implants were in tooth bounded saddle, which might be a reason for the preservation of the bone level around the implants due to teeth functional loading, which preserves the alveolar process from atrophy.

Bleeding that occurred in the early loaded group when gingival formers were removed is plaqueinduced mucositis, a plaque was evident on the early loaded group gingival formers. The bleeding did not occur in progressively loaded gingival former as they were replaced every two weeks with a new clean, and sterile healing cap.

\section{CONCLUSION}

The outcomes from this pilot study suggest that immediate progressive loading with healing caps seems to have no significant effect on secondary stability of dental implants when compared with the traditional early loading of dental implants who underwent trans-mucosal healing with gingival formers. Transmucosal healing is possible in the posterior maxilla with a very high success rate especially if the implant was inserted with high primary stability, but strict oral hygiene measure is mandatory to avoid plaque accumulation which may lead to mucositis. Progressive loading with a healing cap is not of a great benefit to the patients in terms of bone training, and it might cause pain while replacing healing caps.

\section{REFERENCES}

- $\quad$ Akin-Nergiz, N., Nergiz, I., Schulz, A., Arpak, N., \& Niedermeier, W. (1998). Reactions of peri-implant tissues to continuous loading of osseointegrated implants. American Journal of Orthodontics and Dentofacial Orthopedics : Official Publication of the American Association of Orthodontists, Its Constituent Societies, and the American Board of Orthodontics, 114(3), 292-298. Retrieved from http:// www.ncbi.nlm.nih.gov/pubmed/9743134

- $\quad$ Appleton, R. S., Nummikoski, P. V., Pigno, M. A., Cronin, R. J., \& Chung, K.-H. (2005). A radiographic assessment of progressive loading on bone around single osseointegrated implants in the posterior maxilla. Clinical Oral Implants Research, 16(2), 161-167. https://doi.org/10.1111/j.16000501.2004.01089.x

- Brånemark, P. I., Adell, R., Breine, U., Hansson, B. O., Lindström, J., \& Ohlsson, A. (1969). Intra-osseous anchorage of dental prostheses. I. Experimental studies. Scandinavian Journal of Plastic and Reconstructive Surgery, 3(2), 81-100. Retrieved from http://www.ncbi.nlm.nih.gov/pubmed/4924041

- Brånemark, P. I., Hansson, B. O., Adell, R., Breine, U., Lindström, J., Hallén, O., \& Ohman, A. (1977). Osseointegrated implants in the treatment of the edentulous jaw. Experience from a 10-year period. Scandinavian Journal of Plastic and Reconstructive Surgery. Supplementum, 16, 1-132. Retrieved from http://www.ncbi.nlm.nih.gov/ pubmed/356184

- Cameron, H., Macnab, I., \& Pilliar, R. (1972). Porous surfaced Vitallium staples. South African Journal of Surgery. Suid-Afrikaanse Tydskrif Vir Chirurgie, 10(2), 63-70. Retrieved from http://www.ncbi.nlm.nih.gov/ pubmed $/ 4603120$

- Cameron, H. U., Pilliar, R. M., \& MacNab, I. (1973). The effect of movement on the bonding of porous metal to bone. Journal of Biomedical Materials Research, 7(4), 301-311. https://doi.org/10.1002/jbm.820070404

- Frost, H. M. (1983). The regional acceleratory phenomenon: a review. Henry Ford Hospital Medical Journal, 31(1), 3-9. Retrieved from http://www.ncbi.nlm.nih.gov/ pubmed/6345475

- $\quad$ Ghoveizi, R., Alikhasi, M., Siadat, M.-R., Siadat, H., \& Sorouri, M. (2013). A radiographic comparison of progressive and conventional loading on crestal bone loss and density in single dental implants: a randomized controlled trial study. Journal of Dentistry (Tehran, Iran), 10(2), 
155-163. Retrieved from http://www.ncbi.nlm.nih.gov/ pubmed/23724215

- Gotfredsen, K., Berglundh, T., \& Lindhe, J. (2001). Bone reactions adjacent to titanium implants subjected to static load. A study in the dog (I). Clinical Oral Implants Research, 12(1), 1-8. Retrieved from http://www.ncbi.nlm. nih.gov/pubmed/11168265

- Henry, P., \& Rosenberg, I. (n.d.). Single-stage surgery for rehabilitation of the edentulous mandible: preliminary results. Practical Periodontics and Aesthetic Dentistry: PPAD, 6(9), 15-22; quiz 24. Retrieved from http://www. ncbi.nlm.nih.gov/pubmed/7488750

- Isidor, F. (1996). Loss of osseointegration caused by occlusal load of oral implants. A clinical and radiographic study in monkeys. Clinical Oral Implants Research, 7(2), 143152. https://doi.org/10.1034/j.1600-0501.1996.070208.x

- Johansson, C.; Albrektsson, T. (n.d.). Integration of Screw Implants in the Rabbit: A 1-yr Follow-up of Removal Torque of Titanium Implants. Retrieved from https:// web.a.ebscohost.com/abstract?direct=true $\&$ profile=ehost $\&$ scope $=$ site $\&$ authtype $=$ crawler $\&$ jrnl $=08822786 \& A N=37$ 793307\&h=Kd\%2B2BJVx7rv5kzkWW11VSI69M\%2Fvs P6fDTv3tDKJ\%2Fi0b9GU\%2F7pC5y1TZITXdsDoSipM MGq\%2BzYSFxteP5uIaHCcQ\%3D\%3D\&crl=c\&resultN $\mathrm{s}=$ AdminWebAuth\&resul

- Maniatopoulos, C., Pilliar, R. M., \& Smith, D. C. (1986). Threaded versus porous-surfaced designs for implant stabilization in bone-endodontic implant model. Journal of Biomedical Materials Research, 20(9), 1309-1333. https:// doi.org/10.1002/jbm.820200907
- Melsen, B., \& Lang, N. P. (2001). Biological reactions of alveolar bone to orthodontic loading of oral implants. Clinical Oral Implants Research, 12(2), 144-152. Retrieved from http://www.ncbi.nlm.nih.gov/pubmed/11251664

- $\quad$ Misch, C. E., Hoar, J., Beck, G., Hazen, R., \& Misch, C. M. (1998). A bone quality-based implant system: a preliminary report of stage I \&amp; stage II. Implant Dentistry, 7(1), 35-42. Retrieved from http://www.ncbi.nlm.nih.gov/ pubmed/9588972

- Moncler, S. S., Salama, H., Reingewirtz, Y., \& Dubruille, J. H. (2013). Timing of Loadinghttp://coimplante.odo.br/ Biblioteca/Carga\%20Imediata/Immediate\%20loading\%20 protocol\%20-\%20Tarnow\%20et\%20al.pdf and Effect of Micromotion on Bone - Dental Implant Interface : Review of Experimental Literature. (December).

- Roberts, W. E. (1988). Bone tissue interface. Journal of Dental Education, 52(12), 804-809. Retrieved from http:// www.ncbi.nlm.nih.gov/pubmed/3057027

- Skalak, R. (1983). Biomechanical considerations in osseointegrated prostheses. The Journal of Prosthetic Dentistry, 49(6), 843-848. Retrieved from http://www.ncbi.nlm.nih. gov/pubmed/6576140

- Titanium implants and lateral forces. An experimental study with sheep. - PubMed - NCBI. (n.d.). Retrieved April 15, 2019, from https://www.ncbi.nlm.nih.gov/pubmed/9555205

- $\quad$ Wu, K. K., Guise, E. R., Frost, H. M., \& Mitchell, C. L. (1978). The surgical technique for forequarter amputation: a clinical analysis of ten cases. Orthopedics, 1(6), 457461. https://doi.org/10.3928/0147-7447-19781101-04 